\title{
ON THE SYMMETRIC RANGE ASSIGNMENT PROBLEM IN WIRELESS AD HOC NETWORKS
}

\author{
Douglas M. Blough ${ }^{1}$, Mauro Leoncini ${ }^{2}$, Giovanni Resta ${ }^{3}$, Paolo Santi ${ }^{4}$ \\ ${ }^{1}$ School of ECE, Georgia Institute of Technology, Atlanta GA. \\ ${ }^{2}$ Dip. di Dipartimento di Scienze Sociali, Cognitive e Quantitative, \\ Università di Modena e Reggio Emilia, Italy. \\ ${ }^{3,4}$ Istituto di Informatica e Telematica del CNR, Area della Ricerca di Pisa, Italy. \\ ${ }^{1}$ doug.bloug@ece.gatech.edu, ${ }^{2}$ leoncini@unimo.it, ${ }^{3}$ p.santi@iit.cnr.it, ${ }^{4}$ g.resta@iit.cnr.it
}

\begin{abstract}
In this paper we consider a constrained version of the range assignment problem for wireless ad hoc networks, where the value the node transmitting ranges must be assigned in such a way that the resulting communication graph is strongly connected and the energy cost is minimum. We impose the further requirement of symmetry on the resulting communication graph. We also consider a weaker notion of symmetry, in which only the existence of a set of symmetric edges that renders the communication graph connected is required. Our interest in these problems is motivated by the fact that a (weakly) symmetric range assignment can be more easily integrated with existing higher and lower-level protocols for ad hoc networks, which assume that all the nodes have the same transmitting range. We show that imposing symmetry does not change the complexity of the problem, which remains NP-hard in two and three-dimensional networks. We also show that a weakly symmetric range assignment can reduce the energy cost considerably with respect to the homogeneous case, in which all the nodes have the same transmitting range, and that no further (asymptotic) benefit is expected from the asymmetric range assignment. Hence, the results presented in this paper indicate that weak symmetry is a desirable property of the range assignment.
\end{abstract}

\section{Introduction}

Recent emergence of affordable, portable, wireless communication and computation devices has resulted in the rapid growth of mobile wireless networks. Among these, ad hoc networks, i.e. networks of mobile, untethered units communicating with each other via radio transceivers, are receiving increasing attention in the scientific community. Ad hoc networks can be used wherever a wired backbone is not viable, e.g. in mobile computing applications in areas 
where other types of infrastructures are unavailable, to provide communications during emergencies, or to monitor remote geographical regions.

In ad hoc networks, every node $u$ is characterized by a transmitting range $r_{u}$ : when $u$ sends a message, all the nodes at distance at most $r_{u}$ from $u$ can potentially receive the message. If the recipient is not an immediate neighbor of $u$, the message must be routed to the destination through a multi-hop path. For this reason, ad hoc networks are also called multi-hop packet radio networks.

One of the major concerns in wireless ad hoc networks is reducing node power consumption. In fact, nodes are usually equipped with a limited capacity battery, and battery recharge and/or replacement is very difficult or even impossible in many application scenarios (e.g., wireless sensor networks [23]). Hence, reducing power consumption is often the only way to extend network lifetime. It is known that one of the main sources of power consumption in a wireless node is communication, and that the power $p_{u}$ required by node $u$ to transmit data is related to its transmitting range $r_{u}[10]$. Thus, node transmitting ranges should be set as small as possible consistently with some requirement (e.g., strong connectivity) on the resulting network topology.

Given a transmitting range assignment (range assignment for short) for each node in the network, the communication graph $G$ is defined, where directed edge $(u, v)$ exists in $G$ if and only if $v$ is at distance at most $r_{u}$ from $u$. Although current transceivers and communication protocols are designed for a fixed transmitting range (e.g., 250 meters in the widely used IEEE 802.11 standard [1]), a scenario in which the transmitting range is not fixed is fully compatible with current technology. The range assignment could be decided prior to node deployment in the case of stationary networks when information on the physical node placement are available, or it can be varied dynamically in presence of mobility or when the physical node placement is unknown. Distributed topology control protocols aimed at dynamically changing the transmitting range assignment in order to keep the network connected and minimize energy consumption have been recently presented in $[17,24,26]$.

The problem of assigning transmitting range to nodes in such a way that the resulting communication graph is strongly connected and the energy cost is minimized is called the range assignment problem (RA), and was first studied in [18]. In [18], it is shown that RA for one-dimensional networks (i.e., nodes in a line) is in $\mathrm{P}$, while it is NP-hard in the case of three-dimensional networks. For two-dimensional networks, the problem remains NP-hard [7].

Results concerning a variant of RA in which the range assignment induces a communication graph of diameter at most $h$, for some constant $h$, were also derived in $[7,8,9,18]$. However, we believe this version of the problem is less interesting from a practical point of view. In fact, imposing a topology which is "too connected" would often cause communication interference to occur even between nodes that are far apart, thus decreasing the network capacity. This phenomenon is confirmed by theoretical as well as experimental results $[13,15$, 16 , which show that the communication graph in wireless ad hoc networks should be as sparse as possible, while preserving connectivity. 
A simpler version of RA, in which all the units must have the same transmitting range $r$, was also investigated. We call this problem the homogeneous range assignment problem (HRA). The value of $r$ ensuring connectivity with high probability when nodes are distributed in a given region according to some probability distribution was derived in $[4,14,20,21,22,27]$.

In this paper, we consider $\mathrm{RA}$ with the constraint that the range assignment be symmetric, i.e. such that edge $(u, v)$ is in $G$ if and only if $(v, u)$ is in $G$. We call this problem the symmetric range assignment problem (SRA). We will also investigate a weaker version of the problem, called weak symmetric range assignment (WSRA), in which the requirement for symmetry applies only to a well defined subset of the edges. We are aware of only one paper addressing the symmetric range assignment problem [6], where the authors present a $(1+\ln 2+\epsilon)$ approximation algorithm for a problem equivalent to WSRA. Our interest in studying SRA and WSRA will be clearly motivated in the next section.

First, we show that SRA (and, hence, WSRA) remains NP-hard in two and three-dimensional networks. Hence, imposing (weak) symmetry does not change the complexity of the problem. Then, we investigate the asymptotic cost of the solution of WSRA. We prove that the solutions to RA and WSRA have the same asymptotic cost, thus showing that imposing weak symmetry on the communication graph has only a marginal influence on its energy cost. Finally, we determine bounds on the magnitude of this cost for two typical instances of the problem, i.e. the random instance, in which nodes are distributed uniformly at random in $[0,1]^{d}$, and the $(\Delta, \delta)$-instance, in which the maximum and minimum mutual distances between nodes are $\Delta$ and $\delta$, respectively. The bounds presented in this paper can be compared to similar bounds for different variants of RA obtained in $[4,7,8,9,18]$.

\section{Motivation}

While transceivers with dynamically changing transmitting range are compatible with current technology, most of the existing wireless devices, which are commonly based on either the IEEE 802.11 or the Bluetooth standard, have a fixed transmitting range. As a consequence, most of existing work on routing, clustering and broadcasting protocols for ad hoc networks assume that all the nodes have the same transmitting range $[3,11,25]$.

Observe that routing, broadcasting and clustering protocols are not concerned with the network topology, but they simply assume that transmitting ranges are set in such a way that the resulting communication graph is connected. This means that the presence of an intermediate-level topology control service that dynamically changes node transmitting ranges in order to maintain connectivity while reducing power consumption can be considered. However, due to the homogeneous range assignment assumption, most of the protocols rely (either implicitly or explicitly) on the fact that whenever $u$ sends a message to $v, v$ is capable of communicating directly with $u$, e.g. to acknowledge the message reception. Hence, a topology control mechanism which returns 
a symmetric range assignment is transparent to higher level protocols, which can continue to operate as the range assignment was homogeneous. Further motivations for studying SRA can be found in the full version of the paper [5].

From the discussion above it is clear that the symmetry of the range assignment is a useful property in the design of protocols for wireless ad hoc networks. However, it should be noted that what is really important is the existence of a set of symmetric edges that connect all the nodes in the network. In other words, there could exist further edges for which symmetry is not guaranteed, but removing these edges from the communication graph does not cause disconnection. We call a range assignment with this property weakly symmetric.

It is important to note that while imposing weak symmetry does not impair connectivity, it has a beneficial effect on both node power consumption and network capacity. For a given set of nodes, it can be easily seen that the cost of the optimal symmetric range assignment is higher than that of the weakly symmetric one. Although quantifying the relation between these costs is not immediate, examples can be found in which the cost reduction is considerable. For instance, consider the node placement depicted in Figure 1. For the network to be connected, nodes $u$ and $v_{1}$ must have a transmitting range of at least $n \delta$. If the range assignment must be symmetric, the transmitting range of $v_{n+1}$ must be $n \delta$ too. Hence, all the $v_{i}$ 's, for $i=2, \ldots, n+1$ must have a transmitting range that enables them to reach the farther between node $v_{1}$ and $v_{n+1}$, which is $\Theta(n \delta)$. Assuming that the energy cost is proportional to the square of the transmitting range (see the next section for a definition of the energy cost), we have that the cost of the symmetric range assignment must be $\Theta\left(n^{3} \delta^{2}\right)$. However, if only weak symmetry is required, all the $v_{i}$ 's, for $i=2, \ldots, n+1$, have a transmitting range of $\delta$, and the total cost is $\Theta\left(n^{2} \delta^{2}\right)$.Thus, the saving with respect to the symmetric case is of a factor $\Theta(n)$.

Summarizing, we can say that considering WSRA instead of SRA reflects the requirement for a connected but as sparse as possible communication graph, and it is then fully consistent with the philosophy of wireless ad hoc networks.

\section{Preliminaries}

Let $V=\left\{v_{1}, \ldots, v_{n}\right\}$ be a set of points in the $d$-dimensional Euclidean space. The set $V$ represents the nodes of the network. For any two points $v_{i}, v_{j}$ in $V$, $d\left(v_{i}, v_{j}\right)$ denotes the Euclidean distance between them.

A range assignment for $V$ is a function $R A: V \rightarrow \mathbb{R}^{+}$. Given any range assignment RA for $V$, the communication graph induced by RA is the directed graph $G=(V, E)$, where edge $\left(v_{i}, v_{j}\right) \in E$ if and only if $d\left(v_{i}, v_{j}\right) \leq R A\left(v_{i}\right)$. A range assignment is said to be connecting if the resulting communication graph is strongly connected, and it is said to be symmetric if $\left(v_{i}, v_{j}\right) \in E$ if and only if $\left(v_{j}, v_{i}\right) \in E$. If the range assignment is symmetric, the communication graph can be regarded as undirected, and we are interested in characterizing connectivity instead of strong connectivity. A particular case of symmetric range assignment is the $r$-homogeneous range assignment, defined as $R A\left(v_{i}\right)=r$ for 
$i=1, \ldots, n$, where $r$ is a positive constant. Given a range assignment $R A$ and the corresponding communication graph $G$, we define the symmetric restriction of $G$ as the subgraph $G_{S}$ of $G$ obtained by deleting non-symmetric edges, i.e. $G_{S}=\left(V, E_{S}\right)$, with $E_{S}=\left\{\left(v_{i}, v_{j}\right) \mid\left(d\left(v_{i}, v_{j}\right) \leq R A\left(v_{i}\right)\right) \wedge\left(d\left(v_{i}, v_{j}\right) \leq R A\left(v_{j}\right)\right)\right\}$. A range assignment such that the symmetric restriction of its communication graph is strongly connected is said to be weakly symmetric.

It is known [19] that the power $p_{i}$ required by node $v_{i}$ to correctly transmit data to node $v_{j}$ must satisfy inequality $p_{i} / d\left(v_{i}, v_{j}\right)^{\alpha} \geq \beta$, where $\alpha \geq 1$ is the distance-power gradient and $\beta \geq 1$ is the transmission quality parameter. In ideal conditions we have $\alpha=2$; however, in general it is $1 \leq \alpha \leq 6$ depending on environmental conditions.

Setting $\beta=1$, we can define the cost of a range assignment $R A$ as $c(R A)=$ $\sum_{v_{i} \in V}\left(R A\left(v_{i}\right)\right)^{\alpha}$. We are now ready to formally define the range assignment problems considered in this paper:

Definition 1 Let $V=\left\{v_{1}, \ldots, v_{n}\right\}$ be a set of point in the d-dimensional space:

$R A \quad$ Determine a connecting range assignment $R A$ such that $c(R A)$ is minimum.

WSRA Determine a weakly symmetric range assignment $R A$ such that $c(R A)$ is minimum.

$S R A$ Determine a connecting symmetric range assignment $R A$ such that $c(R A)$ is minimum.

HRA Determine the minimum value of $r$ such that the $r$-homogeneous range assignment is connecting.

In the following, the cost of the solutions of RA, WSRA, SRA and HRA will be denoted $c_{R}, c_{W S}, c_{S}$ and $c_{H R}$, respectively. By definition, it is immediate that $c_{R} \leq c_{W S} \leq c_{S} \leq c_{H R}$.

\section{Complexity of SRA}

Due to space limitations, the proof that SRA (and, consequently, WSRA) for two and three-dimensional networks is NP-hard is not reported. See [5] for details.

\section{Bounds on $c_{W S}$}

In this section we investigate the cost of the optimal solution of WSRA and its relation with the cost of the solutions of other versions of the problem. We start by showing that $c_{R}$ and $c_{W S}$ have the same magnitude.

Given a set $V=\left\{v_{1}, \ldots, v_{n}\right\}$ of points in the $d$-dimensional space, denote with $c_{M S T}$ the cost of a minimum spanning tree on the same set of points, where edge $\left(v_{i}, v_{j}\right)$ has cost $d\left(v_{i}, v_{j}\right)^{\alpha}$. The following theorem is a straightforward consequence of Theorem 3.2 of [18]. For the sake of completeness, we report part of its proof. 
Theorem 1 Let $V=\left\{v_{1}, \ldots, v_{n}\right\}$ be a set of points in the d-dimensional space, for $d=2,3$. For every $\alpha \geq 1$ we have $c_{M S T}<c_{R} \leq c_{W S} \leq 2 c_{M S T}$.

\section{Proof.}

1) $c_{W S} \leq 2 c_{M S T}$. Given the MST $T$ for $V$, we construct a range assignment $R A^{\prime}$ assigning to each node $u$ a transmitting range equal to the $\alpha$-th root of the maximum edge weight among the tree edges incident in $u$. Denote with $G$ and $G_{S}$ the communication graph and the symmetric restriction of the communication graph induced by $R A^{\prime}$. It can be easily seen that every edge of $T$ corresponds to a pair of symmetric edges in $G_{S}$. Hence, $G_{S}$ is connected and $R A^{\prime}$ is a weakly symmetric range assignment. Considering that during the construction of $R A^{\prime}$ each edge of $T$ can be chosen as the "longest" edge, i.e., as transmitting range, by at most two nodes, we have $c_{W S} \leq c\left(R A^{\prime}\right) \leq 2 c_{M S T}$.

2) $c_{M S T}<c_{R}$. See proof of Theorem 3.2 in [18].

Theorem 1 proves that the solutions of RA and WSRA have the same asymptotic cost. Hence, the requirement for weak symmetry has only a marginal effect on the energy cost, while it eases significantly the integration of topology control mechanisms with existing higher and lower-level protocols.

Observe that Theorem 1 states that $c_{M S T}, c_{R}$ and $c_{W S}$ have the same magnitude, but gives no clue on how large this magnitude actually is. In the following sub-sections we evaluate the magnitude of $c_{W S}$ (hence, of $c_{R}$ ) for two typical instances of the range assignment problem.

\subsection{The cost of the random instance}

In the random instance, the network nodes are distributed uniformly at random in $[0,1]^{d}$. In this case, the magnitude of the cost of the Euclidean MST has been evaluated. The following theorem summarizes some results presented in $[2,28,29]$.

Theorem 2 Let $X_{i}, i \geq 1$, be i.i.d. random variables with values in $[0,1]^{d}$, $d \geq 2$, and let $M^{\alpha}\left(X_{1}, \ldots, X_{n}\right)$ be the cost of the MST of the Euclidean graph whose vertices are identified with $X_{1}, \ldots, X_{n}$, and such that for each $1 \leq i \neq$ $j \leq n$ the weight of the edge $e_{i j}$ is $d\left(X_{i}, X_{j}\right)^{\alpha}$. Then for all $d \geq 2$ and $\alpha \geq 1$,

$$
\lim _{n \rightarrow \infty} \frac{M^{\alpha}\left(X_{1}, \ldots, X_{n}\right)}{n^{1-\alpha / d}}=C(\alpha, d)
$$

and

$$
\left|\frac{M^{\alpha}\left(X_{1}, \ldots, X_{n}\right)}{n^{1-\alpha / d}}-C(\alpha, d)\right| \leq \frac{C}{n^{1 / d}},
$$

where $C(\alpha, d)$ denotes a positive constant depending only on $\alpha$ and $d$ and $C$ is a constant.

Combining theorems 1 and 2 we obtain the following tight bound for $c_{W S}$ (and, consequently, for $c_{R}$ ) when nodes are distributed uniformly at random in $[0,1]^{d}$. 
Theorem 3 Let $V=\left\{v_{1}, \ldots, v_{n}\right\}$ be a set of points chosen uniformly and independently at random in $[0,1]^{d}$, for $d=2,3$, and assume $n$ is sufficiently large. Then, for every $\alpha \geq 1$ we have $c_{W S}=\Theta\left(n^{1-\frac{\alpha}{d}}\right)$.

The bound stated in Theorem 3 can be used to compare the magnitude of $c_{W S}$ with the optimal cost of different versions of the range assignment problem in case of random instance. In the following discussion we assume $\alpha=d=2$, since most of existing bounds are for this case. In [20] it is shown that for homogeneous range assignments, the communication graph is connected with high probability if and only if $r=\Omega\left(\sqrt{\frac{\log n}{n}}\right)$. Hence, $c_{H R}=\Theta(\log n)$. When the diameter of the communication graph must be at most $h$, for some positive constant $h$, the cost of the optimal (asymmetric) range assignment is $\Theta\left(n^{1 / h}\right)$ [8]. Thus, while imposing weak symmetry on the range assignment increases its cost of at most a constant factor, the stronger constrains of either homogeneity or small diameter increases the cost significantly, namely of a factor at most $\log n$ in the first case and at most $n^{1 / h}$ in the second. On one hand, these results encourage the utilization of a topology control mechanism to reduce power consumption. On the other hand, they discourage the stronger requirement of small diameter of the communication graph, which causes an increased energy cost and reduces (as discussed in the introduction) the network capacity.

\subsection{The cost of the $(\Delta, \delta)$-instance}

In this sub-section we consider the $(\Delta, \delta)$-instance of WSRA, in which the maximum and minimum mutual distances between nodes in $V$ are $\Delta$ and $\delta$, respectively. Since no results on the cost of the Euclidean MST are known in this case, we recur to a simple recursive construction technique that allows to obtain upper bounds to $c_{W S}$ for $d=2,3$ and $\alpha \geq 1$. These bounds are shown to be tight in some cases.

For the sake of simplicity, we describe the construction for $d=2$. The construction for $d=3$ is an easy modification. Observe that, without loss of generality, we can assume that all the nodes are placed in a square region $S$ whose diagonal is $\Delta$.

Let us begin our construction by dividing $S$ into 4 quadrants. For each quadrant that contains at least one node, we choose one of them as representative. We set the transmitting ranges of these $p_{1} \leq 4$ representatives in such a way that each of them can communicate directly with all the others. For the remaining $n-p_{1}$ nodes, we set the transmitting range to a value sufficient to communicate with the representative in their quadrant. Observe that this construction is weakly symmetric, its cost is at most $p_{1} \Delta^{\alpha}+\left(n-p_{1}\right)(\Delta / 2)^{\alpha}$, and each node is at most 3 hops away from every other.

Let us proceed a step further, subdividing each non-empty quadrant in 4 subquadrants (see Figure 2). Again, in each of the non-empty subquadrants (excluding those containing the $p_{1}$ nodes chosen as representatives in the previous step) we select a node as representative. These $p_{2} \leq 3.4$ nodes will have a transmitting range large enough to communicate with their representative, i.e., 


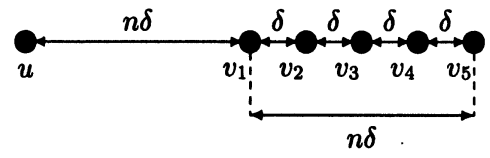

Figure 1. Node placement with consistent cost reduction from the symmetric to the weakly symmetric range assignment. The figure refers to the case of $n=4$.

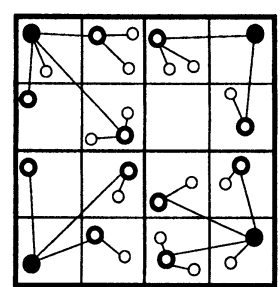

Figure 2. The second step of the construction. For simplicity, we omitted the connections between the first 4 representatives (the black full dots).

at most $\Delta / 2$, while the remaining $\left(n-p_{1}-p_{2}\right)$ nodes can communicate with the representative in their subquadrant with a transmitting range not exceeding $\Delta / 4$. Again, the construction is weakly symmetric, its cost is at most

$$
p_{1} \Delta^{\alpha}+p_{2}\left(\frac{\Delta}{2}\right)^{\alpha}+\left(n-p_{1}-p_{2}\right)\left(\frac{\Delta}{4}\right)^{\alpha},
$$

and each station is at most 5 hops away from every other.

Repeating this construction for $k$ steps, we obtain a weakly symmetric range assignment $R A_{k}$ with cost

$$
c\left(R A_{k}\right) \leq \sum_{i=1}^{k} p_{i}\left(\frac{\Delta}{2^{i-1}}\right)^{\alpha}+\left(n-\sum_{i=1}^{k} p_{i}\right)\left(\frac{\Delta}{2^{k}}\right)^{\alpha}
$$

and each station is at most $1+2 k$ hops away from every other. This construction can be easily applied to the case $d=3$, by means of recursive subdivisions in $2^{3}=8$ subcubes instead of $2^{2}=4$ subquadrants.

In general we have $p_{1} \leq 2^{d}$, and $p_{i} \leq\left(2^{d}-1\right) \cdot\left(2^{d}\right)^{i-1}$ for every $i>1$, hence, by simple manipulation we obtain

$$
c\left(R A_{k}\right) \leq \Delta^{\alpha}+\left(2^{d}-1\right) \Delta^{\alpha} \sum_{i=0}^{k-1} \frac{2^{d i}}{2^{\alpha i}}+\left(n-\sum_{i=1}^{k} p_{i}\right)\left(\frac{\Delta}{2^{k}}\right)^{\alpha} .
$$

Let $t$ be an integer such that $2^{t}>\Delta / \delta$. At step $t$ each subquadrant has thus a diagonal smaller than $\delta$ and contains at most one node. Hence, at step $t+1$ all the nodes will be chosen as representative, i.e., $n=\sum_{i=1}^{t+1} p_{i}$, and the last term of (1) will vanish. So, letting $k^{\prime}=\left\lceil\log _{2}(\Delta / \delta)\right\rceil+1$ we have

$$
c\left(R A_{k^{\prime}}\right) \leq \Delta^{\alpha}+\left(2^{d}-1\right) \Delta^{\alpha} \sum_{i=0}^{k^{\prime}-1} \frac{2^{d i}}{2^{\alpha i}} .
$$

In general the ratio $\Delta / \delta$, and thus the number of recursive steps, can be arbitrarily large. However, it is easily seen that if $\Delta / \delta>n^{1 / d}$, then a weakly 
symmetric range assignment with a smaller cost can be obtained by stopping the construction at step $k^{\prime \prime}=\left(\log _{2} n\right) / d$. In fact, by $\left(n-\sum_{i} p_{i}\right) \leq n$, we obtain:

$$
c\left(R A_{k}^{\prime \prime}\right) \leq \Delta^{\alpha}+\left(2^{d}-1\right) \Delta^{\alpha} \sum_{i=0}^{k^{\prime \prime}-1} \frac{2^{d i}}{2^{\alpha i}}+n\left(\frac{\Delta}{2^{k^{\prime \prime}}}\right)^{\alpha} .
$$

Considering the asymptotic values of $c\left(R A_{k}^{\prime}\right)$ and $C\left(R A_{k}^{\prime \prime}\right)$ in the three cases $\alpha<d, \alpha=d$ and $\alpha>d$ we obtain:

$$
c_{W S}= \begin{cases}O\left(\Delta^{\alpha}\right) & \text { if } d<\alpha \\ O\left(\Delta^{\alpha} \min \{\log (\Delta / \delta), \log n\}\right) & \text { if } d=\alpha \\ O\left(\Delta^{\alpha} \min \left\{(\Delta / \delta)^{d-\alpha}, n^{1-\frac{\alpha}{\alpha}}\right\}\right) & \text { if } d>\alpha\end{cases}
$$

Observing that in general $\Delta / \delta=\Omega\left(n^{1 / d}\right)$ holds, the bounds on $c_{W S}$ can be rewritten as

$$
c_{W S}=\left\{\begin{array}{lll}
O\left(\Delta^{\alpha}\right) & \text { if } & d<\alpha \\
O\left(\Delta^{\alpha} \log n\right) & \text { if } & d=\alpha \\
O\left(\Delta^{\alpha} n^{1-\frac{\alpha}{d}}\right) & \text { if } & d>\alpha
\end{array}\right.
$$

The upper bounds established in equation (2) can be compared with the trivial lower bound of $\Omega\left(n \delta^{\alpha}\right)$ on the cost of any connecting range assignment for the $(\Delta, \delta)$-instance of the problem. Observe that this trivial lower bound cannot be improved, since it can actually be achieved when points are located in a lattice of step $n^{1 / d}$. Hence, the construction presented above in general is not optimal. However, in the case of well spread instances, i.e. when $\Delta / \delta=$ $\Theta\left(n^{1 / d}\right)$, our construction is optimal when $d>\alpha$ (for example, when $d=3$ and $1 \leq \alpha<3$ ). In fact, in this case the lower bound can be rewritten as $\Omega\left(\Delta^{\alpha} n^{1-\frac{\alpha}{d}}\right)$, which matches the cost of our construction when $d>\alpha$. It should also be observed that when the instance is well spread and $\alpha=d=2$, an optimal asymptotic cost can be achieved using the construction based on the MST described in the proof of Theorem 1. This follows by results presented in $[12,28]$, where it is proved that given $n$ points in $[0,1]^{2}$, there exists a spanning tree $T$ such that $\sum_{\left(v_{i}, v_{j}\right) \in T}\left(d\left(v_{i}, v_{j}\right)\right)^{2} \leq 2 \sqrt{2}$. Scaling by a factor $\Delta$, we have that the weakly symmetric range assignment obtained as described in the proof of Theorem 1 has cost $O\left(\Delta^{2}\right)$, which matches the lower bound. Unfortunately, the methods used in $[12,28]$ to derive these results depend heavily on geometric properties on the plane, hence they do not extend immediately to the case $\alpha=d=3$. Our discussion can be summarized in the following theorem.

Theorem 4 Let $V=\left\{v_{1}, \ldots, v_{n}\right\}$ be a set of points in the d-dimensional space, for $d=2,3$, corresponding to a well spread $(\Delta, \delta)$-instance of WSRA. Then

$$
c_{W S}=\left\{\begin{array}{lll}
\Theta\left(\Delta^{\alpha}\right) & \text { if } & d=\alpha=2 \\
\Theta\left(\Delta^{\alpha} n^{1-\frac{\alpha}{d}}\right) & \text { if } & d>\alpha
\end{array}\right.
$$

Consider now the $(\Delta, \delta)$-instance of WSRA in which the nodes are concentrated at opposite corners of a cube of diagonal $\Delta$. It is immediate that 
connectivity is achieved if and only if at least two nodes have a $\Theta(\Delta)$ transmitting range. Hence, we have $c_{W S}=\Omega\left(\Delta^{\alpha}\right)$, and the bound (2) is optimal in the worst-case when $d<\alpha$.

In a certain sense, the bounds for $c_{W S}$ presented here extends those presented in [8] for the version of RA in which the diameter of the communication graph must be at most $h$, denoted $\mathrm{RA}_{h}$ in the following. The authors only considered the case $\alpha=d=2$, and they show that the cost $c_{R_{h}}$ of the solution of $\mathrm{RA}_{h}$ for a $(\Delta, \delta)$-instance is $\Omega\left(\delta^{2} n^{1+1 / h}\right)$, for any positive constant $h$. They also present a construction which yields a solution of cost $O\left(\Delta^{2} n^{1 / h}\right)$, which is optimal in the case of well-spread instances. Observe that our construction indeed yields a communication graph of diameter $O(\log n)$, hence it can be seen as a generalization of the construction of [8] to the case of $h=O(\log n)$. Furthermore, we cover also the case $d=3$ and different combinations of the values $\alpha$ and $d$. When $\alpha=d=2$, our construction yields a solution of cost $O\left(\Delta^{2} \log n\right)$, which is smaller (as expected) than the cost $O\left(\Delta^{2} n^{1 / h}\right)$ needed when the diameter must be constant. This indicates that also in the case of the $(\Delta, \delta)$-instance the diameter of the communication graph can be traded off with the energy cost.

Finally, we observe that when $d>\alpha$ (as it is likely to be for three-dimensional networks) and the instance is well spread, $c_{W S}$ and $c_{R_{h}}$ have the same magnitude for $h=O(\log n)$. This follows easily by the fact that our construction, which is optimal for well spread instances of WSRA when $d>\alpha$, produces a range assignment of diameter $O(\log n)$. Hence, for well spread instances (and when $d>\alpha$ ), a $O(\log n)$ diameter (instead of $O(n)$ ) comes with no additional (asymptotic) cost.

\section{Conclusions}

In this paper we have studied the impact of imposing the (weak) symmetry constraint to the range assignment problem for wireless ad hoc networks. We have shown that the requirement for symmetry (hence, for weak symmetry) does not change the complexity of the problem, which remains NP-hard for two and three-dimensional networks. We have also shown that the solutions of RA and WSRA have the same asymptotic cost. This means that the requirement for weak symmetry have small impact on the energy cost of the optimal solution. We have also determined bounds on the magnitude of the solution of WSRA for two typical instances of the problem, i.e. the random instance, that accounts for those situations in which node positions are not known in advance or may change with time, and the $(\Delta, \delta)$-instance, that accounts for the case in which at least partial information on node positions are available.

In summary, the results presented in this paper have shown that a weakly symmetric range assignment can reduce the energy cost considerably with respect to the homogeneous case, and that no further (asymptotic) benefit is expected from the asymmetric range assignment. Thus, the goal of a "good" topology control mechanism should be to provide a weakly symmetric range assignment, rather than an asymmetric range assignment as in the case of ex- 
isting protocols. On the other hand, a stronger requirement on the diameter (constant or logarithmic in $n$ ) of the communication graph would increase the energy cost significantly while reducing the network capacity. However, when $d>\alpha$ a communication graph with diameter $O(\log n)$ is achievable with no additional (asymptotic) cost if the instance is well spread.

Observe that, due to the complexity of the problem, only heuristic approaches can be considered. In this perspective, the relation between WSRA and MST can be very useful in driving the design of a topology control protocol that returns a good approximation of the optimal solution. The design of a distributed weakly symmetric topology control mechanism is one of the most important problems left open.

Finally, establishing the relation between the cost of the solution to WSRA and SRA remains open.

\section{References}

[1] Wireless LAN Medium Access Control and Physical Layer Specifications, Aug. 1999. IEEE 802.11 Standard (IEEE Computer Society LAN MAN Standards Committee).

[2] D. Aldous, J.M. Steele, "Asymptotics for Euclidean Minimal Spanning Trees on Random Points", Probab. Theory Relat. Fields, Vol. 92, pp. 247-258, 1992.

[3] S. Basagni, D. Bruschi, I. Chlamtac, "A Mobility-Transparent Deterministic Broadcast Mechanism for Ad Hoc Networks", IEEE Transactions on Networking, Vol. 7, n. 6, pp. 799-807, 1999.

[4] D.M. Blough, P. Santi, "The Random Point Graph Model for Ad Hoc Networks and its Application to the Range Assignment Problem", Tech. Rep. IMC-B4-0105, Istituto di Matematica Computazionale del CNR, Pisa - Italy, Dec. 2001.

[5] D.M. Blough, M. Leoncini, G. Resta, P. Santi, "On the Symmetric Range Assignment Problem in Wireless Ad Hoc Networks", Tech. Rep. IMC-B4-01-07, Istituto di Matematica Computazionale del CNR, Pisa - Italy, Nov. 2001.

[6] G. Calinescu, I.I. Mandoiu, A. Zelikovsky, "Symmetric Connectivity with Minimum Power Consumption in Radio Networks", to appear in Proc. 2nd IFIP Conf. on Theoretical Computer Science, Montreal, August 2002.

[7] A.E.F. Clementi, P. Penna, R. Silvestri, "Hardness Results for the Power Range Assignment Problem in Packet Radio Networks", Proc. 2nd International Workshop on Approximation Algorithms for Combinatorial Optimization Problems (RANDOM/APPROX '99), LNCS (1671), pp. 197-208, 1999.

[8] A.E.F. Clementi, P. Penna, R. Silvestri, "The Power Range Assignment Problem in Radio Networks on the Plane", Proc. XVII Symposium on Theoretical Aspects of Computer Science (STACS 00), LNCS (1770), pp. 651-660, 2000.

[9] A.E.F. Clementi, A. Ferreira, P. Penna, S. Perennes, R. Silvestri, "The Minimum Range Assignment Problem on Linear Radio Networks", Proc. 8th European Symposium on Algorithms (ESA 2000), LNCS (1879), pp. 143-154, 2000.

[10] L.M. Feeney, M. Nilson, "Investigating the Energy Consumption of a Wireless Network Interface in an Ad Hoc Networking Environment", Proc. IEEE INFOCOM 2001, pp. 1548-1557, 2001. 
[11] M. Gerla, J. Tzu-Chen Tsai, "Multicluster, Mobile, Multimedia Radio Network", Wireless Networks, Vol. 1, pp. 255-265, 1995.

[12] E.N. Gilbert, H.O. Pollak, "Steiner Minimal Trees", SIAM J. Appl. Math., Vol. 16, pp. 1-29, 1968.

[13] M. Grossglauser, D. Tse, "Mobility Increases the Capacity of Ad Hoc Wireless Networks", Proc. IEEE INFOCOM 2001, pp. 1360-1369, 2001.

[14] P. Gupta, P.R. Kumar, "Critical Power for Asymptotic Connectivity in Wireless Networks", Stochastic Analysis, Control, Optimization and Applications, Birkhauser, Boston, pp. 547-566, 1998.

[15] P. Gupta, P.R. Kumar, "The Capacity of Wireless Networks", IEEE Trans. Information Theory, Vol. 46, n. 2, pp. 388-404, 2000.

[16] J. Li, C. Blake, D.S.J. De Couto, H. Imm Lee, R. Morris, "Capacity of Ad Hoc Wireless Networks", Proc. ACM MOBICOM 2001, pp. 61-69, 2001.

[17] L. Li, J.H. Halpern, P. Bahl, Y. Wang, R. Wattenhofer, "Analysis of a ConeBased Distributed Topology Control Algorithm for Wireless Multi-hop Networks", Proc. ACM PODC 2001, 2001.

[18] L.M. Kirousis, E. Kranakis, D. Krizanc, A. Pelc, "Power Consumption in Packet Radio Networks", Theoretical Computer Science, Vol. 243, pp. 289-305, 2000.

[19] K. Pahlavan, A. Levesque, Wireless Information Networks, John Wiley and Sons, New York, 1995.

[20] P. Panchapakesan, D. Manjunath, "On the Transmission Range in Dense Ad Hoc Radio Networks", Proc. IEEE SPCOM 2001, 2001.

[21] T.K. Philips, S.S. Panwar, A.N. Tantawi, "Connectivity Properties of a Packet Radio Network Model", IEEE Trans. Information Theory, Vol. 35, n. 5, pp. 10441047, 1989.

[22] P. Piret, "On the Connectivity of Radio Networks", IEEE Trans. Information Theory, Vol. 37, n. 5, pp. 1490-1492, 1991.

[23] G.J. Pottie, W.J. Kaiser, "Wireless Integrated Network Sensors", Communications of the ACM, Vol. 43, n. 5, pp. 51- 58, 2000.

[24] R. Ramanathan, R. Rosales-Hain, "Topology Control of Multihop Wireless Networks using Transmit Power Adjustment", Proc. IEEE Infocom 2000, pp. 404$413,2000$.

[25] S. Ramanathan, M. Steenstrup, "A Survey of Routing Techniques for Mobile Communication Networks", Mobile Networks and Applications, Vol. 1, n. 2, pp. 89-104, 1996.

[26] V. Rodoplu, T.H. Meng, "Minimum Energy Mobile Wireless Networks", IEEE Journal Selected Areas in Comm., Vol. 17, n. 8, pp. 1333-1344, 1999.

[27] P. Santi, D.M. Blough, F. Vainstein, "A Probabilistic Analysis for the Range Assignment Problem in Ad Hoc Networks", Proc. ACM MobiHoc01, pp. 212-220, 2001.

[28] J.M. Steele, "Growth Rates of Euclidean Minimal Spanning Trees with Power Weighted Edges", Annals of Probability, Vol. 16, pp. 1767-1787, 1988.

[29] J.E. Yukich, "Asymptotics for Weighted Minimal Spanning Trees on Random Points", Stochastic Processes and their Appl., Vol. 85, pp. 123-128, 2000. 